\title{
Direct detection of Higgs-portal dark matter at the LHC
}

\author{
Abdelhak Djouadi ${ }^{1,2, a}$, Adam Falkowski ${ }^{1, \mathrm{~b}}$, Yann Mambrini ${ }^{1, \mathrm{c}}$, Jérémie Quevillon ${ }^{1, \mathrm{~d}}$ \\ ${ }^{1}$ Laboratoire de Physique Théorique, Université Paris-Sud, 91405 Orsay, France \\ ${ }^{2}$ CERN, 1211, Geneva 23, Switzerland
}

Received: 22 February 2013 / Revised: 30 April 2013 / Published online: 14 June 2013

(C) The Author(s) 2013. This article is published with open access at Springerlink.com

\begin{abstract}
We consider the process in which a Higgs particle is produced in association with jets and show that monojet searches at the LHC already provide interesting constraints on the invisible decays of a $125 \mathrm{GeV}$ Higgs boson. Using the latest monojet search performed by the CMS collaboration with $4.7 \mathrm{fb}^{-1}$ of data, we set the $95 \%$ confidence level limit on the invisible Higgs decay rate to be less than the total Higgs rate in the Standard Model. This limit could be significantly improved when more data at higher center of mass energies are collected, provided systematic errors on the Standard Model contribution to the monojet background can be reduced. In the context of Higgs portal models of dark matter, we then discuss how the LHC limits on the invisible Higgs branching fraction impose strong constraints on the dark matter scattering cross section on nucleons probed in direct detection experiments.
\end{abstract}

\section{Introduction}

The existence of a boson with a mass around $M_{H}=$ $125 \mathrm{GeV}$ is now firmly established [1,2]. The observed properties of the new particle are consistent with those of the Standard Model (SM) Higgs boson. ${ }^{1}$ Nevertheless, it is conceivable that the Higgs particle may have other decay channels that are not predicted by the SM. Determining or constraining non-standard Higgs boson decays will provide a vital input to model building beyond the SM.

A very interesting possibility that is often discussed is a Higgs boson decaying into stable particles that do not interact with the detector. Common examples where Higgs particles can have invisible decay modes include decays into

\footnotetext{
${ }^{1}$ See for example [3-12].

${ }^{a}$ e-mail: abdelhak.djouadi@th.u-psud.fr

b e-mail: adam.falkowski@th.u-psud.fr

${ }^{\mathrm{c}}$ e-mail: yann.mambrini@th.u-psud.fr

d e-mail: jeremie.quevillon@th.u-psud.fr
}

the lightest supersymmetric particle ${ }^{2}$ or decays into heavy neutrinos in the SM extended by a fourth generation of fermions. ${ }^{3}$ In a wider context, the Higgs boson could be coupled to the particle that constitutes all or part of the dark matter in the universe. In these so-called Higgs portal models [17-27] the Higgs boson is the key mediator in the process of dark matter annihilation and scattering, providing an intimate link between Higgs hunting in collider experiments and the direct search for dark matter particles in their elastic scattering on nucleons. In fact, the present LHC Higgs search results, combined with the constraints on the direct detection cross section from the XENON experiment [28], severely constrain the Higgs couplings to dark matter particles and have strong consequences on invisible Higgs decay modes for scalar, fermionic or vectorial dark matter candidates [29].

At the LHC, the main channel for producing a relatively light SM-like Higgs boson is the gluon-gluon fusion (ggF) mechanism. At leading order (LO), the process proceeds through a heavy top quark loop, leading to a single Higgs boson in the final state, $g g \rightarrow H$ [30]. A next-to-leading order (NLO) in perturbative QCD, an additional jet can be emitted by the initial gluons or the internal heavy quarks, leading to $g g \rightarrow H g$ final states [31-34] (additional contributions are also provided by the $g q \rightarrow H q$ process). As the QCD corrections turn out to be quite large, the rate for $H+1$ jet is not much smaller than the rate for $H+0$ jet. The next-to-nextto-leading order (NNLO) QCD corrections [35-39], besides significantly increasing the $H+0$ and $H+1$ jet rates, lead to $H+2$ jet events. The latter event topology also occurs at LO in two other Higgs production mechanisms: vector boson fusion (VBF) $q q \rightarrow H q q$ and Higgs-strahlung $(\mathrm{VH})$ $q \bar{q} \rightarrow H W / H Z \rightarrow H q \bar{q}$ which have rather distinct kinematical features compared to the gluon fusion process; for a review, see Ref. [13, 14].

\footnotetext{
${ }^{2}$ For reviews on Higgs physics, see e.g. [13, 14].

${ }^{3}$ Note that in view of the recent LHC data, a perturbative SM with a 4 th generation fermions is excluded; see $[15,16]$.
} 
Hence, if the Higgs boson is coupled to invisible particles, it may recoil against hard QCD radiation, leading to monojet events at the LHC. The potential of monojets searches to constrain the invisible decay width of a light Higgs boson has been pointed out before [40, 41]. In this paper we update and extend these analyses. We place constraints on the Higgs invisible rate defined as

$R_{\mathrm{inv}}^{\mathrm{pp}}=\frac{\sigma(p p \rightarrow H) \times \mathrm{BR}(H \rightarrow \mathrm{inv} .)}{\sigma(p p \rightarrow H)_{\mathrm{SM}}}$.

We will argue that the existing monojet searches at the LHC [42, 43] yield the constraint $R_{\text {inv }}^{\mathrm{pp}} \lesssim 1$. The constraint is much better than expected. Indeed, early studies [44-50], focusing mainly on the VBF production channel, concluded that observation of invisible Higgs decays was only possible at the highest LHC energy, $\sqrt{s}=14 \mathrm{TeV}$, and with more than $10 \mathrm{fb}^{-1}$ data. Bounds on invisible Higgs based on the $1 \mathrm{fb}^{-1}$ monojet search in ATLAS [51] were studied in Ref. [40, 41], where a weaker limit of $R_{\text {inv }}^{\text {pp }} \lesssim 4$ was obtained for $M_{h} \sim 125 \mathrm{GeV}$.

One one hand, the constraint at the level $R_{\mathrm{inv}}^{\mathrm{pp}} \sim 1$ means that the monojet searches cannot yet significantly constrain the invisible Higgs branching fraction if the production rate of the $125 \mathrm{GeV}$ Higgs boson is close to the SM one. In fact, in that case much stronger constraints follow from global analyses of the visible Higgs decay channels, which disfavor $\operatorname{BR}(H \rightarrow$ inv. $)>0.2$ at $95 \%$ confidence level (CL) $[3,4]$. However, in models beyond the SM, the Higgs production rate may well be enhanced, and in that case the monojet constraints discussed here may become relevant. In this sense, our results are complementary to the indirect constraints on the invisible branching fraction obtained by measuring visible Higgs decays.

In the next step, we discuss the connection between the Higgs invisible branching fraction and the direct dark matter detection cross section. We work in the context of Higgs portal models and consider the cases of scalar, fermionic and vectorial dark matter particles (which we generically denote by $\chi$ ) coupled to the Higgs boson. To keep our discussion more general, the Higgs $-\chi \chi$ couplings are not fixed by the requirement of obtaining the correct relic density from thermal history. ${ }^{4}$ In each case, the LHC constraint $\mathrm{BR}(H \rightarrow$ inv. $)$ can be translated into a constraint on the Higgs boson couplings to the dark matter particles. We will show that these constraints are competitive with those derived from the XENON bounds on the dark mat-

\footnotetext{
${ }^{4}$ Instead, we assume that one of the multiple possible processes (e.g. co-annihilation, non-thermal production, $s$-channel poles of particles from another sector) could arrange that the dark matter relic abundance is consistent with cosmological observations.
}

ter scattering cross section on nucleons. ${ }^{5}$ We discuss how future results from invisible Higgs searches at the LHC and from direct detection experiments will be complementary in exploring the parameter space of Higgs portal models.

The rest of this letter is organized as follows. In the next section, we present our analysis of invisible Higgs production at the LHC. We estimate the sensitivity to the invisible Higgs rate of the CMS monojet search using $4.7 \mathrm{fb}^{-1}$ of data at $\sqrt{s}=7 \mathrm{TeV}$ [42]. We also study the constraints from the recent ATLAS monojet search using $10 \mathrm{fb}^{-1}$ of data at $\sqrt{s}=8 \mathrm{TeV}$ [43]. In the following section we discuss the interplay of the monojet constraints on the invisible Higgs decays and the indirect constraints from the global analysis of the LHC Higgs data. We show that a portion of the theory space with a large Higgs invisible branching fraction favored by global fits is excluded by the monojet constraints. We then move on to discuss the implications for Higgs portal dark matter models and the complementarity between dark matter direct detection at the LHC and in XENON. In the last section we present short conclusions.

\section{Monojet constraints on the invisible width}

In this section we estimate the sensitivity of current monojet searches at the LHC to a Higgs particles that decays invisibly. We rely on the searches for monojets performed by CMS using $4.7 \mathrm{fb}^{-1}$ of data at $7 \mathrm{TeV}$ center of mass energy [42]. The basic selection requirements used by CMS are as follows:

- at least one jet with $p_{T}^{j}>110 \mathrm{GeV}$ and $\left|\eta^{j}\right|<2.4$;

- at most two jets with $p_{T}^{j}>30 \mathrm{GeV}$;

- no isolated leptons.

A second jet with $p_{T}^{j}$ above $30 \mathrm{GeV}$ is allowed provided it is not back-to-back with the leading one, $\Delta \phi\left(j_{1}, j_{2}\right)<$ 2.5. Incidentally, this is advantageous from the point of view of invisible Higgs searches, as Higgs production at the LHC is often accompanied by more than one jet; vetoing the second jet would reduce the signal acceptance by a factor of $\sim 2$. The CMS collaboration quotes the observed event yields and expected SM background for four different cuts on the missing transverse momentum: $p_{T}^{\text {miss }}>250$, $300,350,400 \mathrm{GeV}$. These events are largely dominated by the SM backgrounds, namely $Z+$ jets, where the $Z$ boson decays invisibly, and $W+$ jets, where the $W$ boson decays leptonically and the charged lepton is not reconstructed. In

\footnotetext{
${ }^{5}$ We note that the process $g g \rightarrow H \rightarrow \chi \chi$ for dark matter $\chi$ production at the LHC is an important component of the (crossed) process for dark matter scattering on nucleons, $g \chi \rightarrow g \chi$ [52].
} 
particular, with $4.7 \mathrm{fb}^{-1}$ data, the CMS collaboration estimates the background to be $7842 \pm 367$ events for $p_{T}^{\text {miss }}>$ $250 \mathrm{GeV}$.

A Higgs boson produced with a significant transverse momentum and decaying to invisible particles may also contribute to the final state targeted by monojet searches. In Fig. 1, we show the fraction of Higgs events produced at the parton level in the $\mathrm{ggF}$ and $\mathrm{VBF}$ processes with $p_{T}^{\text {Higgs }}$ above a given threshold, assuming $M_{H}=125 \mathrm{GeV}$. One observes that about $0.5 \%$ of ggF events are produced with $p_{T}^{\text {Higgs }}>250 \mathrm{GeV}$, while for the VBF production processes that fraction is larger by a factor of $\sim 3$. In $4.7 \mathrm{fb}^{-1}$ data at $\sqrt{s}=7 \mathrm{TeV}$ this corresponds to about 500 events, assuming the SM production cross sections. This suggests that if an invisible Higgs boson is produced with rates that are comparable or larger than that of the SM Higgs boson, the monojet searches may already provide meaningful constraints.

In order to estimate the sensitivity of the CMS monojet search to the invisible Higgs signal, we generated the $p p \rightarrow H+$ jets $\rightarrow$ invisible + jets process. We used the program POWHEG $[53,54]$ for the ggF and VBF channels at the parton level, and Madgraph 5 [55] for the VH channels. Showering and hadronization was performed using Pythia 6 [56] and Delphes 1.9 [57] was employed to simulate the CMS detector response. We imposed the analysis cuts listed above on the simulated events so as to find the signal efficiency. As a cross-check, we passed $(Z \rightarrow v v)+$ jets background events through the same simulation chain, obtaining efficiencies consistent within $15 \%$ with the data-driven estimates of that background provided by CMS.

The signal event yield depends on the cross section in each Higgs production channel and on the Higgs branching

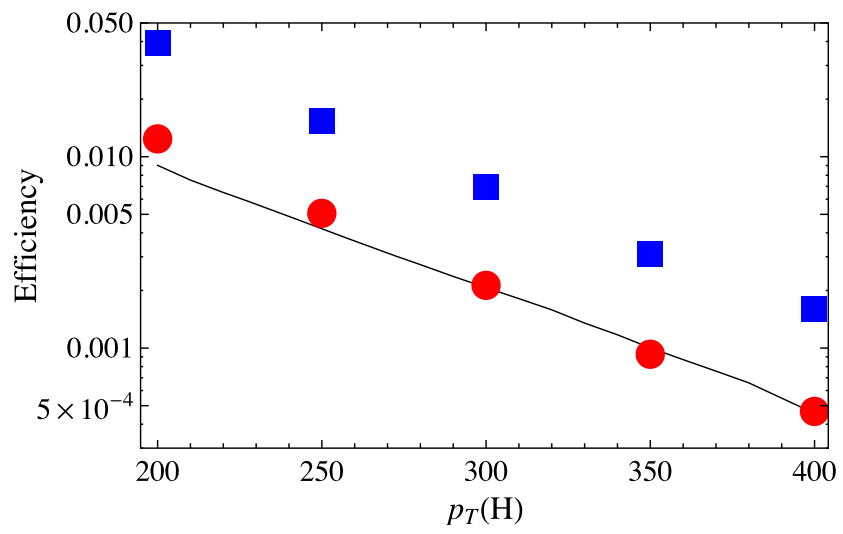

Fig. 1 The fraction of events with Higgs transverse momentum above a given threshold for the ggF (red circles) and VBF (blue squares) production modes. The distributions were obtained at NLO using the program POWHEG [53]. In the case of ggF, the simulations included the finite quark mass effects [54], and we find good agreement with the NNLO distribution obtained using the program HRes [39] (black line) (Color figure online) fraction into invisible final states. Thus, strictly speaking, the quantities that are being constrained by the CMS search $\operatorname{are}^{6} R_{\mathrm{inv}}^{\mathrm{gg}}$ and $R_{\mathrm{inv}}^{\mathrm{V}}$ defined as

$R_{\mathrm{inv}}^{\mathrm{gg}}=\frac{\sigma(g g \rightarrow H) \times \mathrm{BR}(H \rightarrow \mathrm{inv})}{\sigma(g g \rightarrow H)_{\mathrm{SM}}}$,

$R_{\mathrm{inv}}^{\mathrm{V}}=\frac{[\sigma(q q \rightarrow H q q)+\sigma(q \bar{q} \rightarrow V H)] \times \mathrm{BR}(H \rightarrow \mathrm{inv})}{\sigma(q q \rightarrow H q q)_{\mathrm{SM}}+\sigma(q \bar{q} \rightarrow V H)_{\mathrm{SM}}}$

Currently available data do not allow us to independently constrain $R_{\mathrm{inv}}^{\mathrm{gg}}$ and $R_{\mathrm{inv}}^{\mathrm{V}}$. Thus, for the sake of setting limits, we assume that the proportions of ggF, VBF and $\mathrm{VH}$ rates are the same as in the SM, and we take the inclusive cross sections to be $\sigma(g g \rightarrow H)_{\mathrm{SM}}=15.3 \mathrm{pb}, \sigma(q q \rightarrow$ $H q q)_{\mathrm{SM}}=1.2 \mathrm{pb}$ and $\sigma(q \bar{q} \rightarrow H V)_{\mathrm{SM}}=0.9 \mathrm{pb}[58,59]$. With this assumption, after the analysis cuts the signal receives about $30 \%$ contribution from the VBF and VH production modes, and the rest from ggF; thus CMS constrains the combination $R_{\mathrm{inv}}^{\mathrm{pp}} \approx \frac{2}{3} R_{\mathrm{inv}}^{\mathrm{gg}}+\frac{1}{3} R_{\mathrm{inv}}^{\mathrm{V}}$.

Our results are presented in Table 1 . We display the predicted event yields $N_{\text {inv }}^{\mathrm{gg}}, N_{\text {inv }}^{\mathrm{V}}$ in, respectively, the ggF and $\mathrm{VBF}+\mathrm{VH}$ channels for the four CMS $p_{T}^{\text {miss }}$ cuts. ${ }^{7}$ For convenience, we also reproduce the expected $\Delta N_{95 \%}^{i, \text { exp }}$ and observed $\Delta N_{95 \%}^{\mathrm{obs}} 95 \% \mathrm{CL}$ limits on the number of extra non-SM events quoted by CMS in Ref. [42] for each cut. Comparing $N_{\mathrm{inv}}^{\mathrm{gg}}+N_{\mathrm{inv}}^{\mathrm{V}}$ with $\Delta N_{95} \%$ it is straightforward to obtain $95 \%$ CL expected and observed limits on $R_{\text {inv }}^{\mathrm{pp}}$ corresponding to each cut reported in Table 1 . We find the best expected limit $R_{\mathrm{inv}}^{\mathrm{pp}} \leq 2.1$ for the $p_{T}^{\text {miss }} \geq 250 \mathrm{GeV}$ cut. The observed limit is better than the expected one thanks to an $\mathcal{O}(1 \sigma)$ downward fluctuation of the SM background, and we find $R_{\mathrm{inv}}^{\mathrm{pp}} \leq 1.6$ at $95 \% \mathrm{CL}$ for that cut. A stronger limit on $R_{\mathrm{inv}}^{\mathrm{pp}}$ can be derived by binning the number of events given in Table 1 into exclusive bins, and then

Table 1 Limits on the on the invisible Higgs rate $R_{\text {inv }}^{\mathrm{pp}}$. The event yields are given for each reported $p_{T}^{\text {miss }}$ cut of the CMS monojet search, separately for the $\mathrm{ggF}$ and $\mathrm{VBF}+\mathrm{VH}$ production modes, assuming the SM Higgs production cross sections in these channels and $\mathrm{BR}(H \rightarrow$ inv $)=100 \%$. We also give the expected and observed $95 \%$ CL limits on the number of non-SM events reported by CMS [42], which allow us to derive $95 \%$ CL expected and observed limits on $R_{\mathrm{inv}}^{\mathrm{pp}}$

\begin{tabular}{lrrllll}
\hline$p_{T}^{\text {miss }}[\mathrm{GeV}]$ & $N_{\mathrm{inv}}^{\mathrm{gg}}$ & \multicolumn{1}{c}{$N_{\mathrm{inv}}^{\mathrm{V}}$} & $\Delta N_{95 \%}^{\exp }$ & $\Delta N_{95 \%}^{\text {obs }}$ & $\exp . R_{\text {inv }}^{\mathrm{pp}}$ & obs. $R_{\text {inv }}^{\mathrm{pp}}$ \\
\hline 250 & 250 & 110 & 779 & 600 & 2.1 & 1.6 \\
300 & 110 & 50 & 325 & 368 & 2.1 & 2.3 \\
350 & 46 & 25 & 200 & 158 & 2.8 & 2.2 \\
400 & 22 & 13 & 118 & 95 & 3.4 & 2.7 \\
\hline
\end{tabular}

${ }^{6}$ Assuming custodial symmetry, $R_{\mathrm{inv}}^{\mathrm{VH}}=R_{\mathrm{inv}}^{\mathrm{VBF}} \equiv R_{\mathrm{inv}}^{\mathrm{V}}$.

${ }^{7}$ Note that we did not consider the theoretical uncertainties on the cross sections $[58,59]$ and the efficiencies of the $p_{T}$ cuts which, although significant, are currently smaller than the experimental ones. 
combining exclusion limits from all four $p_{T}^{\text {miss }}$ bins. Assuming Gaussian errors, one can recast the limits on the number of non-SM events as $\Delta N^{i}=\Delta N_{0}^{i} \pm \Delta N_{1 \sigma}^{i}$, with $\Delta N_{0}^{i}=\Delta N_{95 \%}^{i, \text { obs }}-\Delta N_{95 \%}^{i, \exp }, \Delta N_{1 \sigma}^{i}=\Delta N_{95 \%}^{i, \exp } / 1.96$, where $i=1 \ldots 4$ indexes the $p_{T}^{\text {miss }}$ bins. The invisible Higgs width would produce an excess of events $\delta N^{i}\left(R_{\mathrm{inv}}^{\mathrm{pp}}\right)$ in all the bins. Assuming in addition small correlations between the errors in various bins, we can thus construct a global $\chi^{2}$ function, $\chi^{2}=\sum_{i}\left[\Delta N_{0}^{i}-\delta N^{i}\left(R_{\mathrm{inv}}^{\mathrm{pp}}\right)\right]^{2} /\left[\Delta N_{1 \sigma}^{i}\right]^{2}$ so as to constrain $R_{\text {inv }}^{\mathrm{pp}}$. Using this procedure we obtain

$R_{\text {inv }}^{\mathrm{pp}} \leq 1.10$ at $95 \% \mathrm{CL}$.

Following the same procedure, we can also constrain separately $R_{\mathrm{inv}}^{\mathrm{gg}}$ and $R_{\mathrm{inv}}^{\mathrm{V}}$, assuming only the $\mathrm{ggF}$ or only the $\mathrm{VBF}+\mathrm{VH}$ Higgs production mode is present. We find $R_{\text {inv }}^{\mathrm{gg}} \leq 2.0$ (when $\mathrm{VBF}$ and $\mathrm{VH}$ are absent) or $R_{\text {inv }}^{\mathrm{V}} \leq 4.0$ (when $\mathrm{ggF}$ is absent) at $95 \% \mathrm{CL}$.

We also study the impact of the ATLAS monojet search [43] with $10 \mathrm{fb}^{-1}$ at $\sqrt{s}=8 \mathrm{TeV}$. ATLAS defines four search categories: SR1, SR2, SR3, SR4 with similar cuts on the visible jets as discussed above for the CMS case, and with the missing energy cut $p_{T}^{\text {miss }}>120,220,350$, $500 \mathrm{GeV}$, respectively. In Table 2 we give the $95 \% \mathrm{CL}$ limits on the invisible rate deduced from the number monojet events reported by ATLAS for each of these categories. We find the best expected limit $R_{\text {inv }}^{\mathrm{pp}} \leq 1.7$ using the $p_{T}^{\text {miss }} \geq$ $220 \mathrm{GeV}$ cut, while the best observed limit is $R_{\text {inv }}^{\mathrm{pp}} \leq 1.4$ using the $p_{T}^{\text {miss }} \geq 500 \mathrm{GeV}$. Unlike in the CMS case, combining ATLAS exclusion limits from different $p_{T}^{\text {miss }}$ bins does not improve the limit of $R_{\mathrm{inv}}^{\mathrm{pp}}$.

\section{Monojet vs. indirect constraints on invisible decays}

In this section we discuss the interplay between the monojet constraints on the invisible Higgs decays and the indirect constraints from the global analysis of the LHC Higgs data $[60,61]$. Assuming the Higgs is produced with the SM cross section, the monojet constraints on the invisible

Table 2 Predicted event yields $N_{\text {inv }}$ (assuming $\mathrm{BR}(H \rightarrow$ inv) $=$ $100 \%$ ), the $1 \sigma$ background uncertainty $\Delta N_{\mathrm{Bkg}}$, and the expected and observed $95 \% \mathrm{CL}$ limits on the invisible Higgs rate $R_{\mathrm{inv}}^{\mathrm{pp}}$ for each reported missing energy cut in the $8 \mathrm{TeV} 10 \mathrm{fb}^{-1}$ ATLAS monojet search [43]. The event yields are given separately for the ggF and $\mathrm{VBF}+\mathrm{VH}$ production modes, assuming the SM Higgs production cross sections in these channels

\begin{tabular}{lrrrll}
\hline$p_{T}^{\mathrm{miss}}[\mathrm{GeV}]$ & $N_{\text {inv }}^{\mathrm{gg}}$ & $N_{\text {inv }}^{\mathrm{V}}$ & $\Delta N_{\text {Bkg }}$ & exp. $R_{\text {inv }}^{\mathrm{pp}}$ & obs. $R_{\text {inv }}^{\mathrm{pp}}$ \\
\hline 120 & 5694 & 1543 & 12820 & 3.5 & 4.4 \\
220 & 904 & 286 & 1030 & 1.7 & 1.6 \\
350 & 110 & 45 & 171 & 2.2 & 3.3 \\
500 & 15 & 9 & 73 & 6.0 & 1.4 \\
\hline
\end{tabular}

branching fraction are not yet relevant. However, in models beyond the SM the Higgs production rate can be significantly enhanced, especially in the gluon fusion channel. One well known example is the case of the SM extended by the fourth generation of chiral fermions where the $g g \rightarrow H$ cross section is enhanced by an order of magnitude. In that class of models a large invisible width may easily arise due to Higgs decays to the fourth generation neutrinos, in which case the monojet constraints discussed here become very important. More generally, the ggF rate can be enhanced whenever there exist additional colored scalars or fermions whose mass originates (entirely or in part) from electroweak symmetry breaking. In a model-independent way, we can describe their effect on the ggF rate via the effective Higgs coupling to gluons:

$\Delta \mathcal{L}=\frac{c_{g g}}{4} H G_{\mu \nu}^{a} G^{\mu \nu, a}$,

where $c_{g g}$ can take arbitrary real values depending on the number of additional colored species, their masses, their spins, and their couplings to the Higgs. Furthermore, given the small Higgs width in the SM, $\Gamma_{H, \mathrm{SM}} \sim 10^{-5} m_{H}$, a significant invisible width $\Gamma_{H \text {,inv }} \sim \Gamma_{H, \mathrm{SM}}$ may easily arise even from small couplings of the Higgs to new physics, for example to massive neutrinos or to dark matter in Higgs portal models. We parametrize these possible couplings simply via the invisible branching fraction $\mathrm{Br}_{\text {inv }}$, which is allowed to take any value between 0 and 1. In Fig. 2 we plot the best fit region to the LHC Higgs data in the $\mathrm{Br}_{\text {inv }}-c_{g g}$ parameter space. For the SM value $c_{g g}=0$ an invisible branching fraction larger than $\sim 20 \%$ is disfavored at $95 \%$ CL. When $c_{g g}>0$, the global fit admits a larger invisible branching

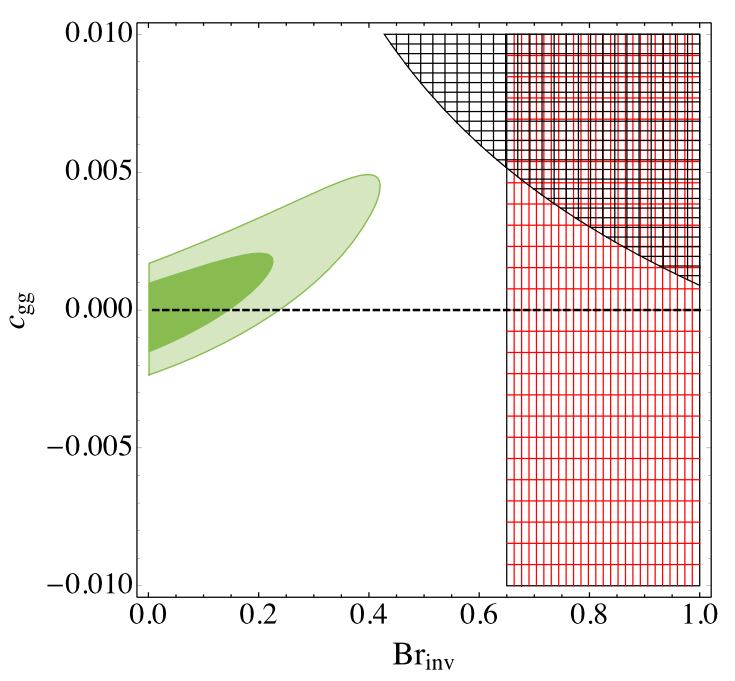

Fig. $268 \%$ CL (light green) and 95\% CL (dark green) best fit regions to the combined LHC Higgs data. The black meshed region is excluded by the monojet constraints derived in this paper, while the red meshed region is excluded by the recent ATLAS $Z+(H \rightarrow$ MET) search [62] (Color figure online) 
fraction, even up to $\mathrm{Br}_{\text {inv }} \sim 50 \%$. Nevertheless, the monojet constraints on the Higgs invisible width derived in this paper are weaker than the indirect constraints from the global fits, when the latest Higgs data are taken into account.

\section{Invisible branching fraction and direct detection}

If the invisible particle into which the Higgs boson decays is a constituent of dark matter in the universe, the Higgs coupling to dark matter can be probed not only at the LHC but also in direct detection experiments. In this section, we discuss the complementarity of these two direct detection methods. We consider generic Higgs-portal scenarios in which the dark matter particle is a real scalar, a real vector, or a Majorana fermion, $\chi=S, V, f$ [29, 63-67]. The relevant terms in the effective Lagrangian in each of these cases are

$$
\begin{aligned}
\Delta \mathcal{L}_{S}= & -\frac{1}{2} m_{S}^{2} S^{2}-\frac{1}{4} \lambda_{S} S^{4}-\frac{1}{4} \lambda_{h S S} H^{\dagger} H S^{2}, \\
\Delta \mathcal{L}_{V}= & \frac{1}{2} m_{V}^{2} V_{\mu} V^{\mu}+\frac{1}{4} \lambda_{V}\left(V_{\mu} V^{\mu}\right)^{2} \\
& +\frac{1}{4} \lambda_{h V V} H^{\dagger} H V_{\mu} V^{\mu}, \\
\Delta \mathcal{L}_{f}= & -\frac{1}{2} m_{f} f f-\frac{1}{4} \frac{\lambda_{h f f}}{\Lambda} H^{\dagger} H f f+\text { h.c. }
\end{aligned}
$$

The partial Higgs decay width into dark matter $\Gamma(H \rightarrow$ $\chi \chi)$ and the spin-independent $\chi$-proton elastic cross section $\sigma_{\chi p}^{\text {SI }}$ can be easily calculated in terms of the parameters of the Lagrangian, and we refer to Ref. [29] for complete expressions. For the present purpose, it is important that both $\Gamma(H \rightarrow \chi \chi)$ and $\sigma_{\chi p}^{\text {SI }}$ are proportional to $\lambda_{H \chi \chi}^{2}$; therefore, the ratio $r_{\chi}=\Gamma(H \rightarrow \chi \chi) / \sigma_{\chi p}^{\text {SI }}$ depends only on the dark matter mass $M_{\chi}$ and known masses and couplings (throughout, we assume the Higgs mass be $M_{H}=125 \mathrm{GeV}$ ). This allows us to relate the invisible Higgs branching fraction to the direct detection cross section:

$\mathrm{BR}_{\chi}^{\mathrm{inv}} \equiv \frac{\Gamma(H \rightarrow \chi \chi)}{\Gamma_{H}^{\mathrm{SM}}+\Gamma(H \rightarrow \chi \chi)}=\frac{\sigma_{\chi p}^{\mathrm{SI}}}{\Gamma_{H}^{\mathrm{SM}} / r_{\chi}+\sigma_{\chi p}^{\mathrm{SI}}}$

with $\Gamma_{H}^{\mathrm{SM}}$ the total decay width into all particles in the SM. For a given $M_{\chi}$, the above formula connects the invisible branching fraction probed at the LHC to the dark matternucleon scattering cross section probed by XENON100. For $m_{p} \ll M_{\chi} \ll \frac{1}{2} M_{H}$, and assuming the visible decay width equals the $\mathrm{SM}$ total width $\Gamma_{H}^{\mathrm{SM}}=4.0 \mathrm{MeV}$ [68], one can write down the approximate relations in the three cases that we are considering,

$$
\begin{aligned}
\mathrm{BR}_{S}^{\mathrm{inv}} \simeq \frac{\left(\frac{\sigma_{S p}^{\mathrm{SI}}}{10^{-9} \mathrm{pb}}\right)}{400\left(\frac{10 \mathrm{GeV}}{M_{S}}\right)^{2}+\left(\frac{\sigma_{S p}^{\mathrm{SI}}}{10^{-9} \mathrm{pb}}\right)}, \\
\mathrm{BR}_{V}^{\mathrm{inv}} \simeq \frac{\left(\frac{\sigma_{V p}^{\mathrm{SI}}}{10^{-9} \mathrm{pb}}\right)}{4 \times 10^{-2}\left(\frac{M_{V}}{10 \mathrm{GeV}}\right)^{2}+\left(\frac{\sigma_{V p}^{\mathrm{SI}}}{10^{-9} \mathrm{pb}}\right)}, \\
\mathrm{BR}_{f}^{\mathrm{inv}} \simeq \frac{\left(\frac{\sigma_{f p}^{\mathrm{SI}}}{10^{-9} \mathrm{pb}}\right)}{3.47+\left(\frac{\sigma_{f p}^{\mathrm{SI}}}{10^{-9} \mathrm{pb}}\right)} .
\end{aligned}
$$

Thus, for a given mass of dark matter, an upper bound on the Higgs invisible branching fraction implies an upper bound on the dark matter scattering cross section on nucleons. In Fig. 3 we show the maximum allowed values of the scattering cross section, assuming the $20 \%$ bound on $\mathrm{BR}_{\chi}^{\text {inv }}$, as follows from indirect constraints on the invisible width discussed in the previous section. Clearly, the relation between the invisible branching fraction and the direct detection cross section strongly depends on the spinorial nature of the dark matter particle, in particular, the strongest (weakest) bound is derived in the vectorial (scalar) case.

In all cases, the derived bounds on $\sigma_{\chi p}^{\mathrm{SI}}$ are stronger than the direct one from XENON100 in the entire range where $M_{\chi} \ll \frac{1}{2} M_{H}$. In other words, the LHC is currently the most sensitive dark matter detection apparatus, at least in the context of simple Higgs-portal models (even more so if $\chi$ is a pseudoscalar, as in [70]). This conclusion does not rely on the assumption that the present abundance of $\chi$ is a thermal relic fulfilling the WMAP constraint of $\Omega_{\mathrm{DM}}=0.226$ [69],

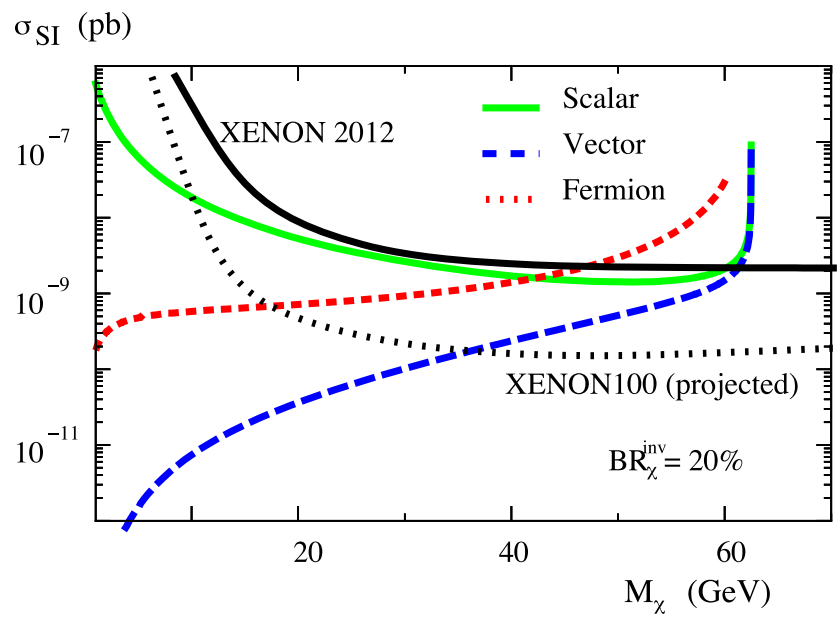

Fig. 3 Bounds on the spin-independent direct detection cross section $\sigma_{x p}^{\text {SI }}$ in Higgs portal models derived for $M_{H}=125 \mathrm{GeV}$ and the invisible branching fraction of $20 \%$ (colored lines). The curves take into account the full $M_{\chi}$ dependence, without using the approximation in Eq. (7). For comparison, we plot the current and future direct bounds from the XENON experiment (black lines) (Color figure online) 
and would only be stronger if $\chi$ constitutes only a fraction of dark matter in the universe. We also compared the bounds to the projected future sensitivity of the XENON100 experiment (corresponding to $60,000 \mathrm{~kg}-\mathrm{d}, 5-30 \mathrm{keV}$ and $45 \%$ efficiency).

Of course, for $M_{\chi}>\frac{1}{2} M_{H}$, the Higgs boson cannot decay into dark matter, ${ }^{8}$ in which case the LHC cannot compete with the XENON bounds.

\section{Conclusions}

We have shown that monojet searches at the LHC already provide interesting limits on invisible Higgs decays, constraining the invisible rate to be less than the total SM Higgs production rate at the $95 \% \mathrm{CL}$. This provides an important constraint on the models where the Higgs production cross section is enhanced and the invisible branching fraction is significant. Monojets searches are sensitive mostly to the gluon-gluon fusion production mode and, thus, they can also probe invisible Higgs decays in models where the Higgs coupling to the electroweak gauge bosons is suppressed. The limits could be significantly improved when more data at higher center of mass energies are collected, provided systematic errors on the Standard Model contribution to the monojet background can be reduced.

We also analyzed in a model-independent way the interplay between the invisible Higgs branching fraction and the dark matter scattering cross section on nucleons, in the context of effective Higgs portal models. The limit $\mathrm{BR}_{\mathrm{inv}}<0.2$, suggested by the combination of Higgs data in the visible channels, implies a limit on the direct detection cross section that is stronger than the current bounds from XENON100, for scalar, fermionic, and vectorial dark matter alike. Hence, in the context of Higgs-portal models, the LHC is currently the most sensitive dark matter detection apparatus.

Acknowledgements The authors would like to thank J. Baglio, E. Bagnaschi, E. Bragina, C. Grojean, P. Boucaud, and T. Volansky as well as the Magic Monday Journal Club for discussions. This work was supported by the French ANR TAPDMS ANR-09-JCJC-0146 and the Spanish MICINN's Consolider-Ingenio 2010 Programme under grant Multi-Dark CSD2009-00064. A.D. thanks the CERN TH unit for hospitality and support.

Open Access This article is distributed under the terms of the Creative Commons Attribution License which permits any use, distribution, and reproduction in any medium, provided the original author(s) and the source are credited.

\footnotetext{
${ }^{8}$ In this case, one should consider the pair production of dark matter particles through virtual Higgs boson exchange, $p p \rightarrow H^{*} X \rightarrow \chi \chi X$. The rates are expected to be rather small [29].
}

\section{References}

1. G. Aad et al. (ATLAS Collaboration), Phys. Lett. B 716, 1 (2012). arXiv:1207.7214 [hep-ex]

2. S. Chatrchyan et al. (CMS Collaboration), Phys. Lett. B 716, 30 (2012). arXiv:1207.7235 [hep-ex]

3. G. Belanger, B. Dumont, U. Ellwanger, J.F. Gunion, S. Kraml, arXiv:1212.5244 [hep-ph]

4. D. Carmi, A. Falkowski, E. Kuflik, T. Volansky, J. Zupan, J. High Energy Phys. 1210, 196 (2012). arXiv:1207.1718 [hep-ph]

5. M. Montull, F. Riva, J. High Energy Phys. 1211, 018 (2012). arXiv:1207.1716 [hep-ph]

6. D. Carmi, A. Falkowski, E. Kuflik, T. Volansky, arXiv:1202.3144 [hep-ph]

7. A. Azatov, R. Contino, J. Galloway, arXiv:1202.3415 [hep-ph]

8. J.R. Espinosa, C. Grojean, M. Muhlleitner, M. Trott, arXiv:1202.3697 [hep-ph]

9. J. Ellis, T. You, arXiv:1204.0464 [hep-ph]

10. P.P. Giardino, K. Kannike, M. Raidal, A. Strumia, arXiv:1203.4254 [hep-ph]

11. V. Barger, M. Ishida, W.-Y. Keung, arXiv:1203.3456 [hep-ph]

12. C. Englert, T. Plehn, M. Rauch, D. Zerwas, P. Zerwas, Phys. Lett. B 707, 512 (2012). arXiv:1112.3007

13. A. Djouadi, Phys. Rep. 457, 1 (2008). hep-ph/0503172

14. A. Djouadi, Phys. Rept. 459, 1 (2008). hep-ph/0503173

15. A. Djouadi, A. Lenz, arXiv:1204.1252 [hep-ph]

16. E. Kuflik, Y. Nir, T. Volansky, arXiv:1204.1975 [hep-ph]

17. V. Silveira, A. Zee, Phys. Lett. B 161, 136 (1985)

18. J. McDonald, Phys. Rev. D 50, 3637-3649 (1994)

19. C.P. Burgess, M. Pospelov, T. ter Veldhuis, Nucl. Phys. B 619, 709-728 (2001). hep-ph/0011335

20. H. Davoudiasl, R. Kitano, T. Li, H. Murayama, Phys. Lett. B 609, 117 (2005). hep-ph/0405097

21. B. Patt, F. Wilczek, hep-ph/0605188

22. S. Andreas, C. Arina, T. Hambye, F.-S. Ling, M.H.G. Tytgat, Phys. Rev. D 82, 043522 (2010). arXiv:1003.2595 [hep-ph]

23. M. Raidal, A. Strumia, Phys. Rev. D 84, 077701 (2011). arXiv:1108.4903 [hep-ph]

24. X.-G. He, J. Tandean, Phys. Rev. D 84, 075018 (2011). arXiv:1109.1277 [hep-ph]

25. A. Drozd, B. Grzadkowski, J. Wudka, J. High Energy Phys. 1204, 006 (2012). arXiv:1112.2582 [hep-ph]

26. Y. Mambrini, Phys. Rev. D 84, 115017 (2011). arXiv:1108.0671 [hep-ph]

27. Y. Mambrini, arXiv:1112.0011 [hep-ph]

28. E. Aprile et al. (XENON100 Collaboration), Phys. Rev. Lett. 109, 181301 (2012). arXiv:1207.5988 [astro-ph.CO]

29. A. Djouadi, O. Lebedev, Y. Mambrini, J. Quevillon, Phys. Lett. B 709, 65 (2012). arXiv:1112.3299 [hep-ph]

30. H. Georgi, S. Glashow, M. Machacek, D. Nanopoulos, Phys. Rev. Lett. 40, 692 (1978)

31. R.K. Ellis, I. Hinchliffe, M. Soldate, J.J. van der Bij, Nucl. Phys. B 297, 221 (1988)

32. A. Djouadi, M. Spira, P.M. Zerwas, Phys. Lett. B 264, 440 (1991)

33. S. Dawson, Nucl. Phys. B 359, 283 (1991)

34. M. Spira, A. Djouadi, D. Graudenz, P. Zerwas, Nucl. Phys. B 453, 17 (1995)

35. R. Harlander, W. Kilgore, Phys. Rev. Lett. 88, 201801 (2002)

36. C. Anastasiou, K. Melnikov, Nucl. Phys. B 646, 220 (2002)

37. V. Ravindran, J. Smith, W. van Neerven, Nucl. Phys. B 665, 325 (2003)

38. S. Catani, D. de Florian, M. Grazzini, P. Nason, J. High Energy Phys. 0307, 028 (2003)

39. D. de Florian, G. Ferrera, M. Grazzini, D. Tommasini, arXiv:1203.6321 [hep-ph]

40. Y. Bai, P. Draper, J. Shelton, arXiv:1112.4496 [hep-ph] 
41. C. Englert, J. Jaeckel, E. Re, M. Spannowsky, Phys. Rev. D 85, 035008 (2012). arXiv:1111.1719 [hep-ph]

42. S. Chatrchyan et al. (CMS Collaboration), CMS-PAS-EXO-11059. arXiv:1206.5663 [hep-ex]

43. ATLAS-CONF-2012-147

44. D. Choudhury, D. Roy, Phys. Lett. B 322, 368 (1994)

45. O. Eboli, D. Zeppenfeld, Phys. Lett. B 495, 147 (2000)

46. R. Godbole et al., Phys. Lett. B 571, 184 (2003)

47. H. Davoudiasl, T. Han, H.E. Logan, Phys. Rev. D 71, 115007 (2005)

48. A. Alves, Phys. Rev. D 82, 115021 (2010). arXiv:1008.0016 [hep$\mathrm{ph}]$

49. J. Kopp, Y. Tsai, Phys. Rev. D 85, 056011 (2012)

50. D. Cavali et al. For an ATLAS simulation, see hep-ph/0203056

51. ATLAS-CONF-2011-096

52. M. Drees, M. Nojiri, Phys. Rev. D 48, 3483 (1993)

53. S. Alioli, P. Nason, C. Oleari, E. Re, J. High Energy Phys. 0904, 002 (2009). arXiv:0812.0578 [hep-ph]

54. E. Bagnaschi, G. Degrassi, P. Slavich, A. Vicini, J. High Energy Phys. 1202, 088 (2012). arXiv:1111.2854 [hep-ph]

55. J. Alwall, M. Herquet, F. Maltoni, O. Mattelaer, T. Stelzer, J. High Energy Phys. 1106, 128 (2011). arXiv: 1106.0522

56. T. Sjostrand, S. Mrenna, P.Z. Skands, J. High Energy Phys. 0605, 026 (2006). hep-ph/0603175
57. S. Ovyn, X. Rouby, V. Lemaitre, arXiv:0903.2225

58. J. Baglio, A. Djouadi, J. High Energy Phys. 1103, 055 (2011). arXiv:1012.0530

59. S. Dittmaier et al. (LHC Higgs cross section Working Group), arXiv: 1101.0593

60. CMS Collaboration, CMS-HIG-13-005

61. ATLAS Collaboration, ATLAS-CONF-2013-034

62. ATLAS Collaboration, ATLAS-CONF-2013-011

63. T. Hambye, M.H.G. Tytgat, Phys. Lett. B 683, 39 (2010). arXiv:0907.1007 [hep-ph]

64. S. Kanemura, S. Matsumoto, T. Nabeshima, N. Okada, Phys. Rev. D 82, 055026 (2010). arXiv: 1005.5651

65. O. Lebedev, H.M. Lee, Y. Mambrini, Phys. Lett. B 707, 570 (2012). arXiv:1111.4482 [hep-ph]

66. J.F. Kamenik, C. Smith, arXiv:1201.4814 [hep-ph]

67. T. Nabeshima, arXiv: 1202.2673

68. A. Djouadi, J. Kalinowski, M. Spira, Comput. Phys. Commun. 108, 56 (1998). hep-ph/9704448

69. E. Komatsu et al. (WMAP Collaboration), Astrophys. J. Suppl. 192, 18 (2011). arXiv: 1001.4538

70. L. Lopez-Honorez, T. Schwetz, J. Zupan, arXiv:1203.2064 [hep$\mathrm{ph}]$ 